\title{
Comparison of muon hodoscope URAGAN and neutron monitors' data for 2008 - 2014
}

\author{
N.S. Barbashina ${ }^{*}, a$, I.I. Astapov ${ }^{a}$, A.N. Dmitrieva ${ }^{a}$, A.Yu. Konovalova ${ }^{a}$, \\ K.G. Kompaniets ${ }^{a}$, E.S. Melnikov ${ }^{b}$, Yu.N. Mishutina ${ }^{a}$, A.A. Petrukhin ${ }^{a}$, \\ V.V. Shutenko ${ }^{a}$, I.I. Yashin ${ }^{a}$ \\ ${ }^{a}$ National Research Nuclear University MEPhI (Moscow Engineering Physics Institute), \\ Moscow 115409, Russian Federation \\ ${ }^{b}$ Fedorov Institute of Applied Geophysics, Moscow 129128, Russian Federation \\ E-mail: NSBarbashina@mephi.ru
}

\begin{abstract}
The results of the comparison of the data of two neutron monitors "Moscow" and "Apatity" with the muon hodoscope URAGAN data for the period 2008 - 2014 that includes different phases of the solar cycle are presented. The obtained correlations between the two types of detectors are compared with different parameters characterizing the state of the interplanetary space. It is shown that during strong disturbances the sensitivity of both methods is practically the same, but the muon data are less susceptible to statistical fluctuations, which is a definite advantage for the study of various heliospheric processes.
\end{abstract}

The 34th International Cosmic Ray Conference

30 July - 6 August, 2015

The Hague, The Netherlands

${ }^{*}$ Speaker 


\section{Introduction}

Studies of cosmic ray temporal variations on the Earth's surface provide important information on the processes in the heliosphere that cause these variations. Such processes strongly affect the low-energy cosmic rays, therefore the studies are mainly conducted using the neutron flux detected by the neutron monitors (NM) on the Earth's surface. Studies of variations of cosmic-ray muons using the muon hodoscope URAGAN are not less interesting, because muons are sensitive to higher primary cosmic ray energies and muon flux provides more large statistics (in comparison with the neutrons) .

To date, a huge amount of the URAGAN [1] experimental data on the registration of the cosmic ray muon flux variations for a long period from 2008 to 2014 which includes different phases of solar activity has been accumulated. In recent times, the use of data of muon detectors is becoming increasingly popular due to the fact that they provide new information for the predicting of the heliospheric disturbance development [2]. However, according to the opinion of some researchers, the higher energies of primary particles responsible for the muon generation (in comparison with the neutrons) represent a significant drawback for the study of moderate heliospheric disturbances because of the lack of the response in the counting rate of the muon detectors. In this paper, the connection between the neutron flux variations registered by the neutron monitors and the muon flux variations registered by the muon hodoscope URAGAN, including those at the disturbed state of the near-Earth space, are studied.

The results of the analysis of the data of two neutron monitors and the muon hodoscope URAGAN data which register the secondary particles generated by the flux of PCR with different energies are presented in this work. For the comparison of variations of the neutron and muon fluxes, the hourly data are used. Two neutron monitors have been selected for the comparison: Moscow [3] and Apatity [4] neutron monitors. The Moscow neutron monitor (MOSC) is located at the same latitude as the URAGAN and has the same threshold rigidity $(2.45 \mathrm{GV})$. The Apatity neutron monitor (APTY) has a lower threshold rigidity $(0.65 \mathrm{GV})$, but its asymptotic directions of the PCR arrival are similar to the URAGAN. The integral counting rates, the rate of variation of the integral counting rate and the daily average amplitude of CR modulations are analyzed and compared. Particular attention is paid to the comparison of the data of two neutron monitors and the muon hodoscope data during the variation of the characteristics of the solar wind, interplanetary magnetic field and the Earth's magnetosphere.

\section{Comparison of the long-term cosmic ray variations}

For the analysis, the data of neutron monitors (obtained from the Internet resource [5]) corrected for the barometric effect and the URAGAN data corrected for the barometric and temperature effects were used. The counting rate of the neutron monitors represents the number of detected particles per minute (for this period, on average approximately 9500 particles for the MOSC and 7000 particles for the APTY). The counting rate of the URAGAN is measured as the number of particles per 1 second (on average 1400 reconstructed muon tracks for each of three hodoscopes included in the URAGAN). Thus, the integral counting rate of three URAGAN supermodules is almost 30 times higher than the counting rate of neutron monitors (the hodoscope registers about 250,000 muons per minute). Figure 1 shows the temporal variations 
of the hourly average deviations of the integral counting rate of the Moscow neutron monitor, Apatity neutron monitor and muon hodoscope URAGAN.
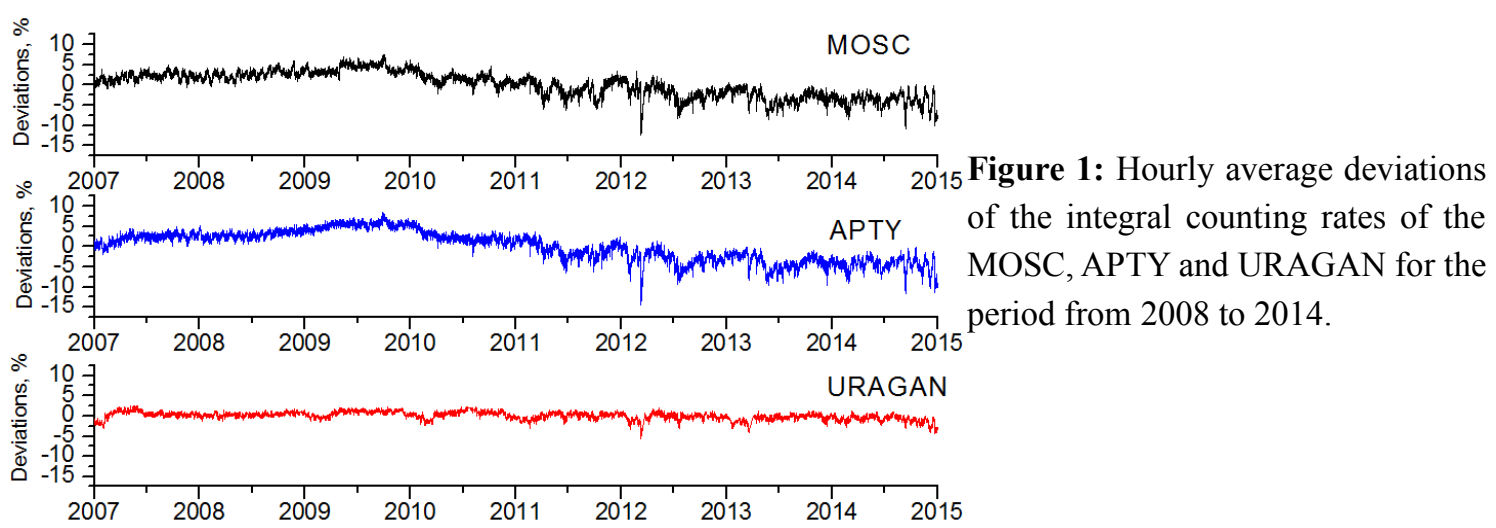

From Figure 1, a trend towards the decrease of the counting rate of neutron monitors due to the increase of solar activity since 2010 is clearly seen. At the same time, a similar effect in the muon counting rate is much weaker according to the URAGAN data: in 2009 the counting rate was maximal, but by the end of the studied period it is only a little less. It is necessary to pay attention to evident decreases of deviations of counting rate in the beginning of each year, which are caused by accumulation of snow in the roof of URAGAN building.

From the point of view of determining quantitative dependences between the integral counting rate of the neutron flux registered by the MOSC and APTY and the muon flux according to the URAGAN data, it is reasonable to consider the correlations between them and to obtain correlation coefficients between each pair of the detectors. The correlation coefficient $(R)$ of the URAGAN with the MOSC and APTY was about 0.6 for the entire period (2008 2014), but by the years it varies considerably. Figure 2 (left) shows the variations of the coefficient $R$ between the normalized integral counting rates of the MOSC and APTY, MOSC and URAGAN, APTY and URAGAN for the each year from 2008 to 2014. Analogously, the correlations between the rates of variation of the neutron monitors and URAGAN counting rates during 1 hour were considered (Fig. 2, right). For the comparison, the sunspot number is shown in Figure 2 [6].
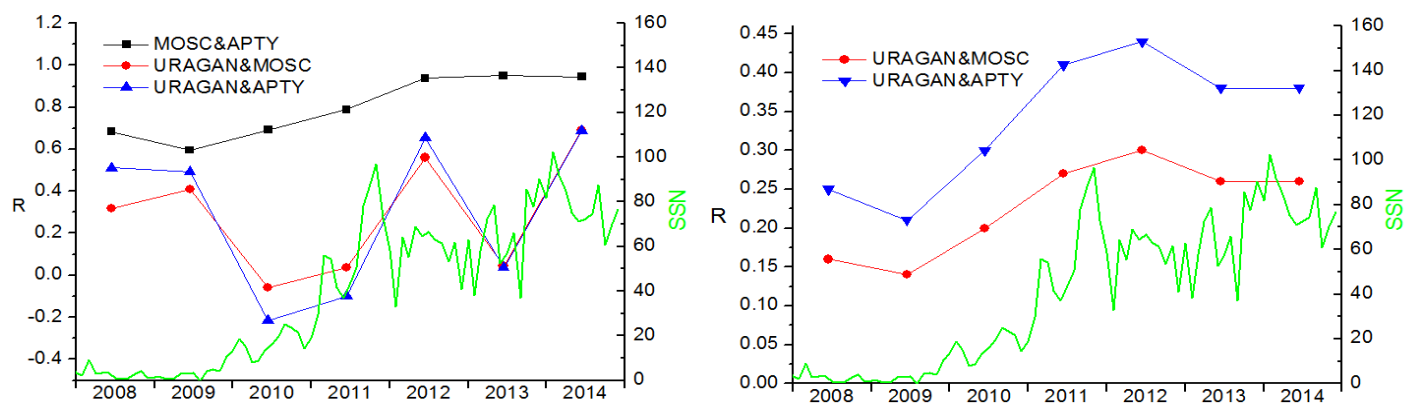

Figure 2: Variations of the coefficients of correlation $R$ between the normalized integral counting rates (left) of the MOSC and APTY (squares), MOSC and URAGAN (circles), APTY and URAGAN (triangles), and correlations coefficients for the rate of variation of their counting rates (right) in the period from 2008 to 2014. The solid line shows the sunspot number (right axes).

Comparison of the normalized integral counting rates showed that the best correlation is observed at the maximum of solar activity: $R_{2012}=0.56$ and $R_{2014}=0.69$ (with the MOSC), 
$R_{2012}=0.65$ and $R_{2014}=0.69$ (with the APTY). The average correlation at the minimum of solar activity is: $R_{2008}=0.32$ and $R_{2009}=0.41$ (with the MOSC), $R_{2008}=0.51$ and $R_{2009}=0.50$ (with the APTY). In 2010, 2011 and 2013 there are practically no correlations. The correlation coefficients for the rate of variation of the counting rate for 1 hour between the URAGAN and NM are significantly lower than in the integral counting rate, but it is noticeable that their time dependences agree well with the variation of the sunspot number.

One more parameter characterizing the cosmic ray variations is the diurnal variation which determines the sensitivity of the detector to different physical processes. Figure 3 shows the yearly average diurnal variation for each detector.
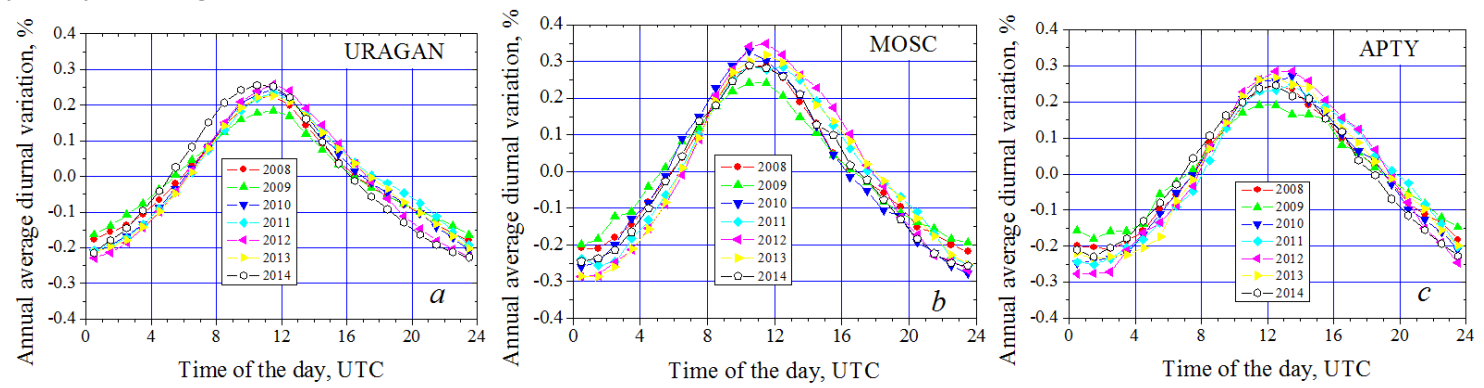

Figure 3: Yearly average diurnal deviations of the integral counting rates of the URAGAN (left), MOSC (center) and APTY (right).

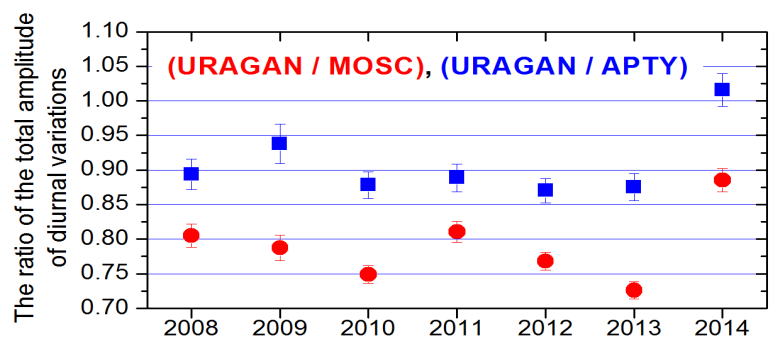

Figure 4: The ratio of the total amplitudes of diurnal variations of the URAGAN to the MOSC (circles) and of the URAGAN to the APTY (squares).

For the convenience of comparison of the amplitudes of diurnal variations, the ratios of the total amplitudes of diurnal variations of the URAGAN to the MOSC and of the URAGAN to the APTY are shown in Figure 4. As is seen from Figure 4, the ratio of the URAGAN to the MOSC is on average about 0.8, and of the URAGAN to the APTY $\sim 0.9$. In 2014, an anomalous behavior of the ratio of the total amplitude of the URAGAN counting rate diurnal variations is observed in comparison with other years: total amplitude of the diurnal variation in the URAGAN is practically the same as in the Apatity and differs from the preceding six years.

\section{Comparison of the data of URAGAN and NMs during the heliospheric disturbances}

Comparison of the URAGAN experimental data with the data of neutron monitors during the heliospheric disturbances in the period from 2008 to 2014 was based on the study of the correlation coefficients of the integral counting rates and their variation rates between the URAGAN and neutron monitors depending on the variation of the parameters of the solar wind (SW), the interplanetary magnetic field (IMF) and the Earth's magnetosphere. Also, the correlation dependences of the monthly averaged diurnal rms-deviations of the data of muon and neutron detectors were compared with the parameters characterizing the solar activity. 

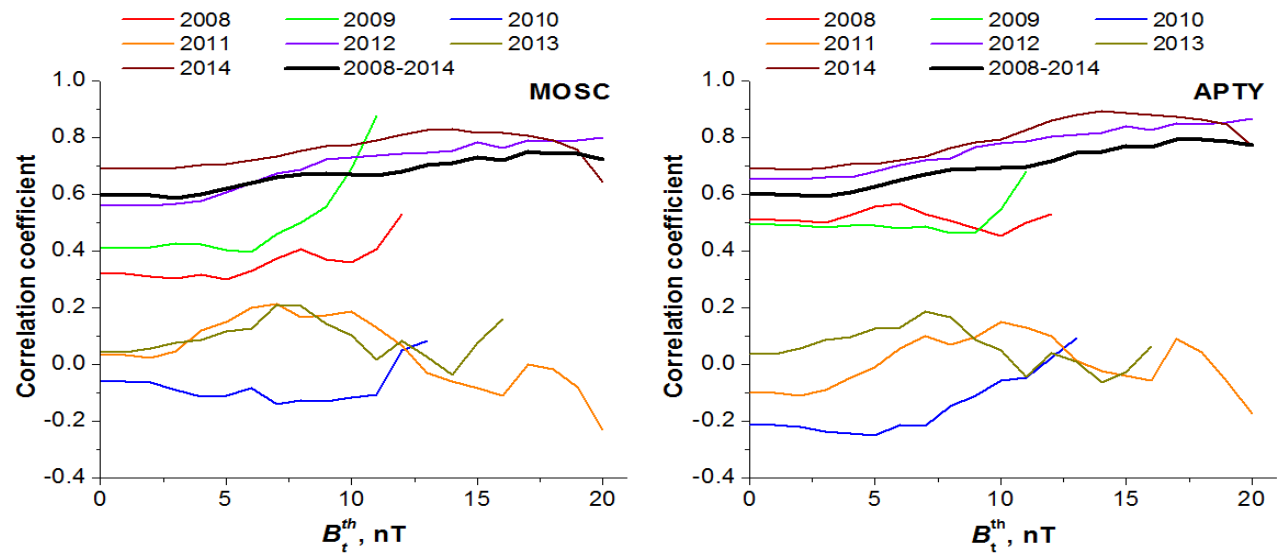

Figure 5: Dependences of the coefficient of correlation of the integral counting rates of the URAGAN with the MOSC (left) and with the APTY (right) on the magnetic induction vector values by years and for the entire period.

For every year separately and for the entire analyzed period, the dependences of the correlation coefficients of the counting rates of neutron monitors with the URAGAN counting rate on the threshold values of the following parameters obtained by the OMNI database [7] were investigated: the solar wind velocity $V_{S W}$, the interplanetary magnetic field induction $B_{t}$ and the geomagnetic index Dst. The minimum values initial for the calculation of correlation coefficients were chosen for each parameter. For example, the minimal value of $B_{t}^{\text {th }}$ is 0 , and every following value is calculated in increments of $1 \mathrm{nT}:\left(B_{t}^{t h}\right)_{i}=\left(B_{t}^{t h}\right)_{\min }+1.0 \cdot i,(i=0,1,2, \ldots)$. For every value of $\left(B_{t}^{\text {th }}\right)_{i}$ (condition that $B_{t} \geqslant\left(B_{t}^{\text {th }}\right)_{i}$ ) the dependences of the URAGAN counting rate on the counting rate of the neutron monitor (the MOSC or the APTY) and the dependences of the rate of the URAGAN counting rate variations per hour on the rate of the neutron monitor (also the MOSC or the APTY) counting rate variations were plotted. If for the current threshold of the magnetic field induction there were at least 30 points, then the linear regression coefficients were determined using the least squares method and the correlation coefficient $R_{i}$ was calculated. The maximum value of the magnetic field intensity, for which the requirement of the minimal number of points had been met, was $20 \mathrm{nT}$. The analysis results are shown in Figures 5 and 6.
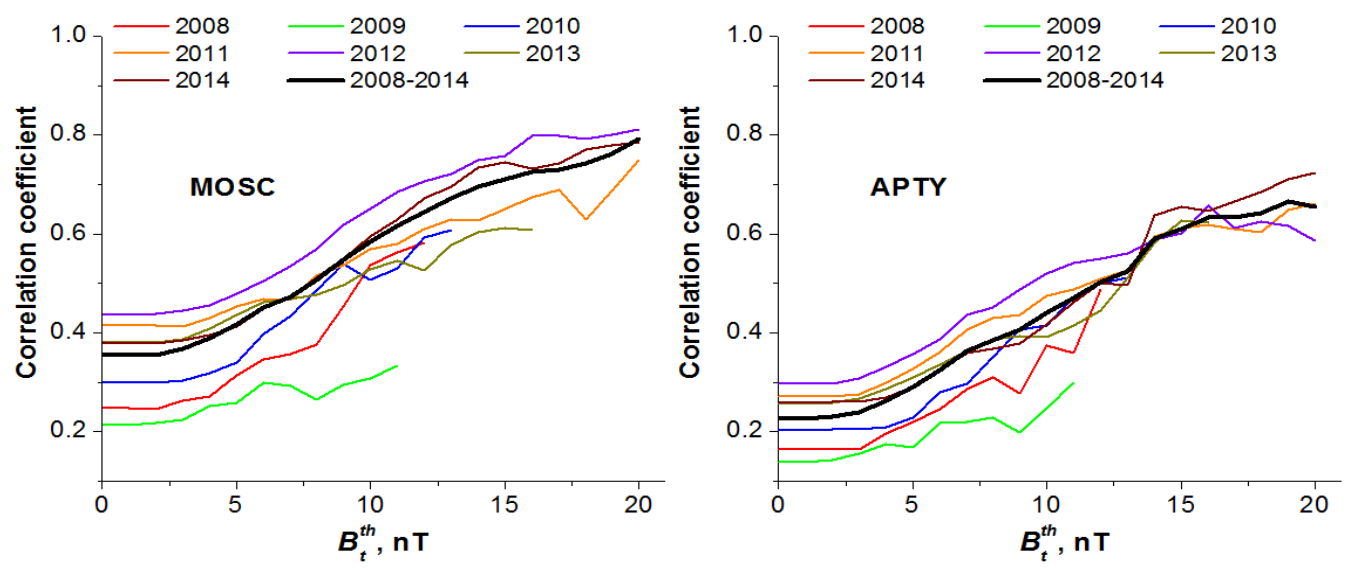

Figure 6: Dependences of the coefficient of correlation of the rate of one-hour variation of the integral counting rates of the URAGAN with the MOSC (left) and with the APTY (right) on the magnetic induction by years and for the entire period. 
Similar studies have been conducted for the solar wind velocity. The results of the analysis for all three quantities ( $V_{S W}, B_{t}$ and $D s t$ ) showed that for the entire period from 2008 to 2014 the correlation coefficients between the integral counting rates of the URAGAN and NM are practically independent of the parameters of the solar wind, IMF and the Earth's magnetic field and lie in the range from 0.6 to 0.95 . Also, no significant differences in the behavior of the coefficients of correlation between the MOSC and URAGAN and between the URAGAN with APTY were found for these quantities. At the same time, the correlation coefficients differ greatly by years. For the entire period, the correlation coefficients of the rates of variation of the integral counting rate of the URAGAN and NM depend strongly on the values of $V_{S W}$, Dst and, especially, $B_{t}$. Correlations of the URAGAN with the APTY are weaker than with the MOSC. Correlations of the URAGAN with both the MOSC and the APTY differ slightly by years.

The plots in figure 7 show the monthly averaged counting rates of the pairs URAGAN with MOSC and URAGAN with APTY (Figures $7 \mathrm{a}$ and $7 \mathrm{~b}$, correspondingly) and the diurnal rms deviations of the same detector pairs (Figures 7c and 7d) for the period from 2008 to 2014. Correlation analysis showed that the correlation coefficient between the monthly averaged values of the counting rate is 0.61 , and the correlation coefficient between monthly averaged diurnal rms deviations is 0.81 . In the second case, the correlation coefficient is significantly higher. Therefore, for the further comparison with the parameters of the near-Earth space (the solar wind velocity, the geomagnetic indices $D s t$ and $K p$, the magnetic induction vector and its projection on the $Z$ axis), the monthly averaged diurnal rms deviations were used. The diurnal rms deviation $\left(\sigma I_{24}\right)$ is calculated according to the following formula:

$$
\sigma I_{24}=\sqrt{\left(\frac{1}{n} \sum_{i=1}^{24} I_{i}^{2}-\left(\frac{1}{n} \sum_{i=1}^{24} I_{i}\right)^{2}\right) \frac{n}{n-1}}
$$
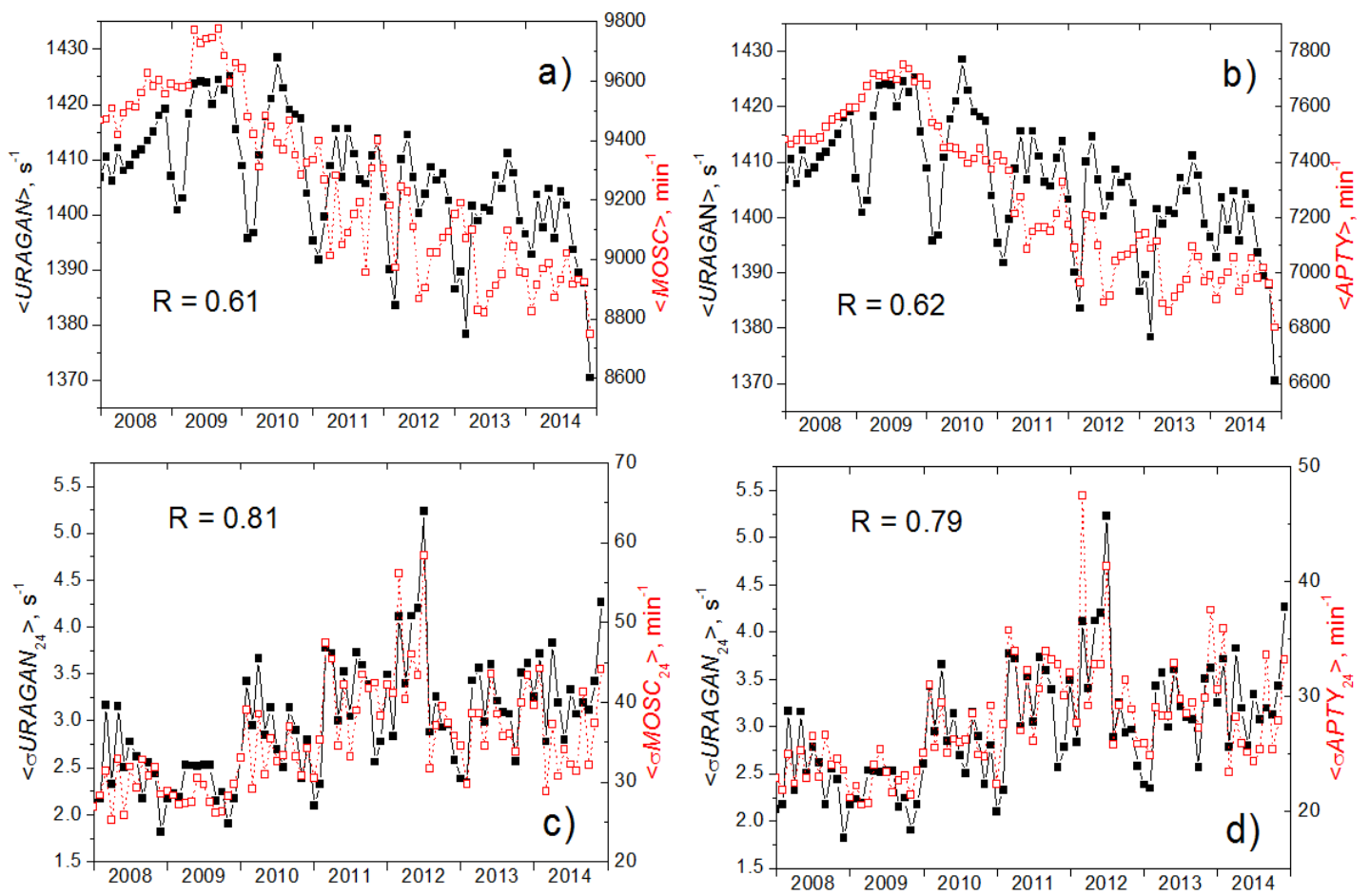

Figure 7: Monthly average values of the counting rate $(a, b)$ and monthly averaged diurnal rms deviations (c, d) of the URAGAN, MOSC and Apatity data (2008 - 2014). 
Study of the correlations between the parameters of solar activity and the URAGAN or neutron monitors separately has a particular interest for the comparison of the muon and neutron fluxes variations. Figure 8 shows the results of comparing of the monthly averaged diurnal rms deviations of the URAGAN data with the solar wind velocity (a), geomagnetic indices Dst (b) and $K p$ (c), the vector of magnetic induction (d) and its projection on the $Z$ axis (e) for the period from 2008 to 2014. In each figure, the correlation coefficient between the analyzed parameters is presented.

Similarly, the monthly averaged rms-deviations of the MOSC and APTY daily data were compared. The results are summarized in Table 1.
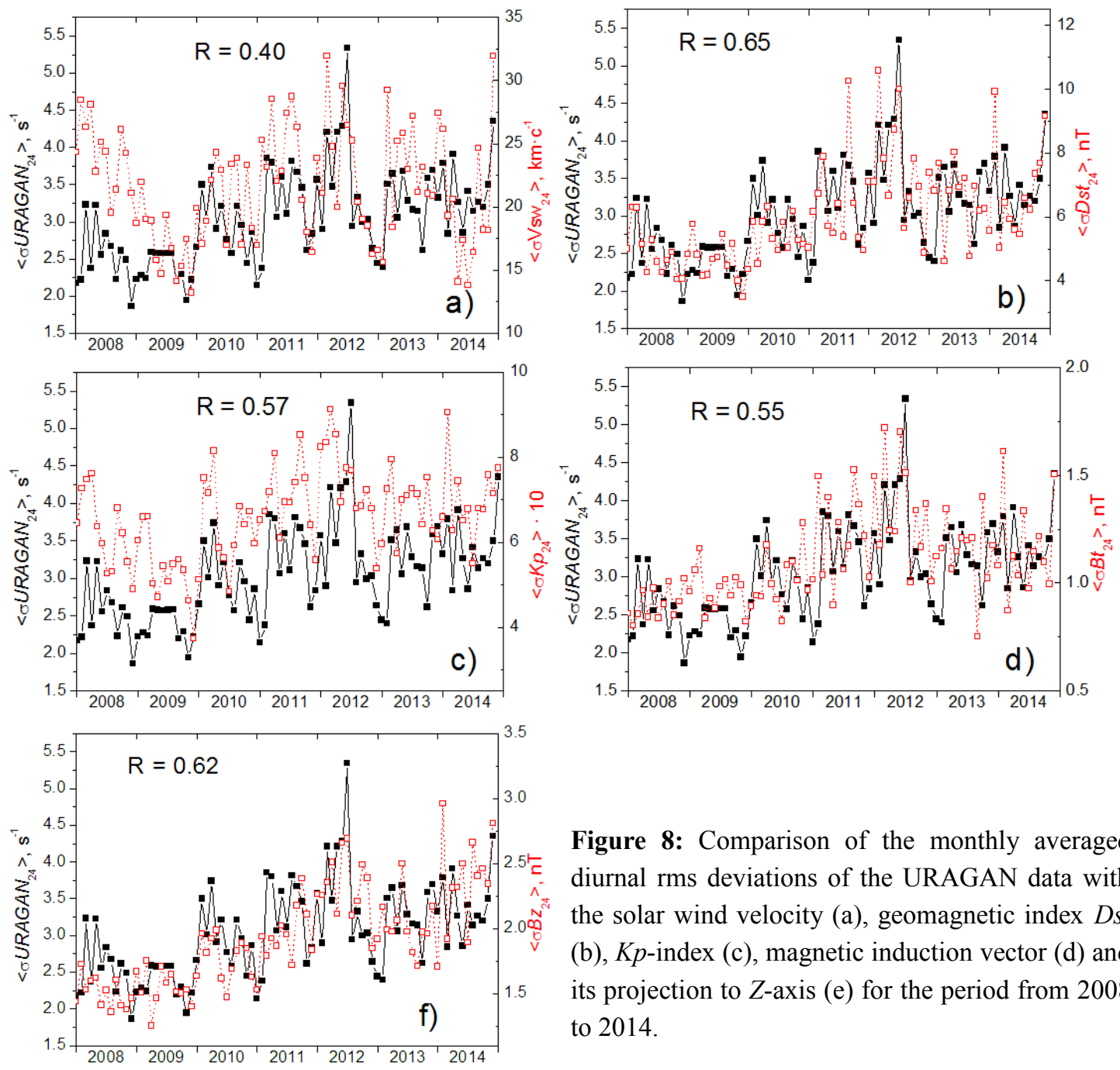

Figure 8: Comparison of the monthly averaged diurnal rms deviations of the URAGAN data with the solar wind velocity (a), geomagnetic index $D s t$ (b), $K p$-index (c), magnetic induction vector (d) and its projection to $Z$-axis (e) for the period from 2008 to 2014 .

Table 1: The correlation coefficients of the monthly averaged diurnal rms deviations of the URAGAN and MOSC data with the solar wind parameters, geomagnetic indices and IMF.

\begin{tabular}{clccccc}
\hline \hline & $\left\langle\sigma V_{S W 24}\right\rangle$ & $\left\langle\sigma D s t_{24}\right\rangle$ & $\left\langle\sigma K p_{24}\right\rangle$ & $\left\langle\sigma B_{t 24}\right\rangle$ & $\left\langle\sigma B_{224}\right\rangle$ \\
\hline$\left\langle\sigma I_{24}\right\rangle$ & URG & 0.40 & 0.65 & 0.57 & 0.55 & 0.62 \\
\hline$\left\langle\sigma I_{24}\right\rangle$ & MOSC & 0.36 & 0.72 & 0.56 & 0.67 & 0.66 \\
\hline$\left\langle\sigma I_{24}\right\rangle$ & APTY & 0.41 & 0.70 & 0.57 & 0.67 & 0.66 \\
\hline \hline
\end{tabular}


The results presented in the table show that the coefficients of the correlations between the variation parameters of muons and neutrons and the characteristics of the solar wind, interplanetary magnetic field and magnetic field of the Earth differ only slightly in spite of the higher primary energy of muons. Using of the monthly averaged diurnal rms deviations of data of the cosmic ray detectors can be effective for the study of long-term variations of the solar activity.

\section{Conclusion}

As a result of comparing the variations of the neutron and muon cosmic ray components, some features have been revealed in the correlation dependences of their characteristics: the anomalous behavior of the total amplitude of the counting rate diurnal variations in 2014 (Figure 4); the significant dependence of the coefficient of correlation between the rates of variation of the counting rate of neutrons and muons on the value of $B_{t}$ (Figure 6); the weaker (in comparison with the parameters of the IMF and geomagnetic activity) connection between the diurnal rms deviations of the counting rate and the solar wind velocity (Figure 8).

\section{Acknowledgements}

The work was performed at the Unique Scientific Facility "Experimental complex NEVOD" with the financial support from the State provided by the Ministry of Education and Science of the Russian Federation (project No. RFMEFI59114X0002).

\section{References}

[1] N.S. Barbashina, R.P. Kokoulin, K.G. Kompaniets, et al. The URAGAN wide-aperture large-area muon hodoscope. Instrum. Exp. Tech., 51, 180-186 (2008).

[2] Y. Okazaki, et al. Drift effects and the cosmic ray density gradient in a solar rotation period: First observation with the Global Muon Detector Network (GMDN), (2008). http://arxiv.org/abs/0802.2312v1

[3] Moscow Neutron Monitor, (2015). http://cosrays.izmiran.ru/main.htm

[4] Apatity Neutron Monitor, (2015). http://pgia.ru/CosmicRay/

[5] Network of Cosmic ray Stations, (2015). http://cr0.izmiran.rssi.ru/common/links.htm

[6] SILSO Database, (2015). http://sidc.oma.be/silso/datafiles

[7] OMNI Database, (2015). http://omniweb.gsfc.nasa.gov/ 